\title{
EFEITOS DO ESTRESSE NO PERÍODO GESTACIONAL EM DIFERENTES MODELOS EXPERIMENTAIS: UMA REVISÃO DA LITERATURA
}

\author{
EFFECTS OF GESTATIONAL STRESS IN DIFERENT EXPERIMENTAL MODELS: A LITERATURE REVIEW
}

\section{Márcio Vinícius Fagundes Donadio ${ }^{1 *}$, Mauro Henrique Moraes Vargas ${ }^{2 *}$}

1mdonadio@pucrs.br, 2moraesvargas@yahoo.com.br *Centro Infantil, Instituto de Pesquisas Biomédicas da Pontifícia Universidade Católica do Rio Grande do Sul (PUCRS).

\section{RESUMO}

Introdução: A exposição a eventos estressantes durante o desenvolvimento gestacional pode induzir a alteraçôes duradouras em diversos sistemas. Objetivo: Avaliar, por meio de uma revisão da literatura, os efeitos do estresse no período gestacional em modelos experimentais e as repercussóes geradas na vida adulta em diferentes sistemas. Materiais e Métodos: Foi pesquisada a base de dados Medline/Pubmed, com a seleçáo de artigos originais em que foi realizado estresse por contençáo em roedores durante o período gestacional, publicados nos últimos dez anos, no idioma inglês, nos quais as seguintes palavras-chave constavam no título ou resumo: prenatal stress (estresse pré-natal), programming (programação) e pregnancy (gravidez), além de suas combinaçôes. Resultados: A maioria dos estudos realizados foi em ratos (91\%), sendo que 56\% utilizaram machos e fêmeas, 35\% utilizaram somente machos e $9 \%$ somente fêmeas. Em relaçáo ao estresse, $65 \%$ realizaram três vezes ao dia e $62 \%$ utilizaram 45 minutos, enquanto outros estudos variaram de 30 minutos até 6 horas. Em $72 \%$ dos estudos o estresse foi inserido na última semana da gestação. No entanto, $40 \%$ dos estudos mostram que os efeitos do estresse no período gestacional influenciaram o desenvolvimento ou alteraram alguma estrutura no sistema nervoso central (SNC), tais como, diminuição da proliferação celular e mudanças na expressão de proteínas no hipocampo, alteraçôes de atividades enzimáticas no hipotálamo etc. Conclusáo: Os resultados indicam que o estresse durante a gestação provoca alteraçóes permanentes em diversos sistemas, principalmente no $\mathrm{SNC}$, nos mecanismos de regulação de comportamentos e da memória. O período gestacional parece ser importante na definição dessas alterações, assim como as diferentes respostas entre machos e fêmeas.

Palavras-chave: Estresse; roedores; gestação; período pré-natal; vida adulta.

\section{ABSTRACT}

Introduction: Exposure to stressful events during the gestational development can induce lasting changes in various systems. Objective: To evaluate, through a literature review, the effects of gestational stress in experimental models on the impact generated in adulthood in different systems. Materials and Methods: We searched the Medline/Pubmed database and selected original articles that studied gestational stress in rodents, which were published in the last ten years, in English. The keywords should appear on the title or summary: prenatal stress, programming and pregnancy and combinations of these. Results: The majority of studies were conducted in rats (91\%), and 56\% used males and females, $35 \%$ used only males and females only $9 \%$. With respect to stress, $65 \%$ conducted three times a day and $62 \%$ used 45 minutes, while other studies ranged from 30 minutes to 6 hours. In $72 \%$ of studies stress was inserted in the last week of pregnancy. However, $40 \%$ of studies show that the effects of gestational stress influenced the development or changed some structure in the central nervous system (CNS), such as, decreased cell proliferation, and 
changes in protein expression in the hippocampus, changes in enzymatic activity in the hypothalamus etc. Conclusion: The results indicate that stress in the gestational period causes permanent changes in many systems, especially in the CNS, in the mechanisms of regulation of behavior and memory. The gestational period appears to be important in defining these changes as well as the different responses between males and females.

Keywords: Stress; rodents; pregnancy; prenatal period; adulthood.

\section{Introdução}

Situações que geram estresse são cada vez mais comuns entre as pessoas e motivaram a condução de muitos estudos para avaliar seus efeitos. O termo "estresse" denota o estado gerado pela percepção de estímulos, internos ou externos, que geram excitaçáo emocional, levando à alteraçáo do estado de homeostase, juntamente com um processo de adaptação que permite ao indivíduo superar certas exigências que o meio ambiente lhe proporciona ${ }^{1,2}$. Estes estímulos ou situaçôes que geram estresse são denominados estressores e a resposta do organismo é a reação ao estresse. Esta resposta compreende aspectos cognitivos, comportamentais e fisiológicos, visando propiciar uma melhor percepção da situação e de suas demandas, o que possibilita uma busca de soluçóes e a seleção de condutas adequadas e a preparaçáo do organismo para agir de maneira rápida e vigorosa ${ }^{3,4}$.

A resposta clássica ao estresse consiste na ativação do sistema simpático e adrenomedular e do eixo hipotálamo-hipófise-adrenal (HPA). É a perturbação do equilíbrio desses sistemas que pode contribuir para a geração de diversos distúrbios. Sob ameaça, o organismo desencadeia uma complexa resposta fisiológica que se inicia no âmbito do sistema nervoso central (SNC), levando a alteraçôes neuroendócrinas, imunológicas, psicomotoras e psicológicas. Esta reação poderá ocasionar modificaçôes importantes na pressão arterial, frequência cardíaca, além de complicações gástricas e respiratórias ${ }^{2,3,5}$.

Diferentes tipos de estímulos resultam em distintos tipos de estresse, tais como o estresse químico, físico, psicológico (ou um conjunto de fatores ambientais e emocionais), entre outros ${ }^{1}$. Dependendo do período da vida humana (ou animal) em que estas situaçóes ocorrem, respostas e consequências específicas serão produzidas. Quando há estresse durante a gestação, no período denominado pré-natal, uma série de repercussóes pode ocorrer para o filhote, tendo em vista que nele se inicia o desenvolvimento e a maturação de diversos sistemas (neurológico, cardiovascular, endócrino etc.) e órgãos essenciais $^{6,7}$. Este processo, conhecido como programming ou programação, é definido com base no conceito de que fatores não genéticos possam agir precocemente e ter repercussóes permanentes na reorganização de sistemas fisiológicos ${ }^{8}$. Novas evidências vêm sendo publicadas nos últimos anos, mostrando que a exposição a eventos estressantes durante o desenvolvimento gestacional pode induzir alterações duradouras em diversas funçôes dos sistemas neuroendócrino e comportamental de animais ${ }^{9-11}$. Estes efeitos persistem durante toda a vida do animal e são acompanhados por alteraçôes na expressão de receptores de hormônios relacionados ao estresse (corticosterona e vasopressina) em diversas regióes do cérebro (hipotálamo, hipocampo e amígdala) ${ }^{12,13}$. Da mesma forma, há mudanças no comportamento aversivo, de medo e ansiedade, aprendizagem e memória na fase adulta ${ }^{14,15}$, além de alteraçôes em parâmetros hemodinâmicos e cardiovasculares, entre outras.

Considerando a frequência das situaçóes de estresse e a importância do período gestacional para o desenvolvimento dos organismos, este estudo tem como objetivo avaliar, por uma revisão na literatura, os efeitos do estresse no período gestacional em modelos experimentais e as repercussóes geradas na vida adulta em diferentes sistemas. Além disso, buscou-se fazer um levantamento dos principais períodos de exposição ao estresse na gestação, assim como as diferentes intensidades de estímulo e suas consequências.

\section{Materiais e métodos}

O presente estudo consiste em uma revisão bibliográfica realizada por meio de uma pesquisa no banco de dados Medline/Pubmed. Foram selecionados somente artigos originais no quais foi inserido estresse por contenção no período gestacional em animais roedores (rato e camundongo), publicados nos últimos dez anos, no idioma inglês, onde as palavras-chave deviam constar no título ou resumo. As palavras-chave utilizadas foram prenatal stress (estresse pré-natal), programming (programação) e pregnancy (gravidez), além de suas combinações. Foram excluídos desta revisão bibliográfica artigos que estudaram outros tipos de estresse durante o período gestacional, outras espécies de animais e também aqueles que não se enquadraram nos objetivos desta revisão. Todos os artigos oriundos das buscas realizadas tiveram seu título e resumo analisados. Em caso de dúvidas sobre a inclusão, o texto completo foi verificado.

Os dados foram analisados a partir da seleçâo dos artigos e da leitura dos resumos e textos e da sistematizaçâo da análise dos métodos e dos principais resultados dos estudos. Para a categorização dos estudos, utilizou-se 
a técnica de análise de conteúdo ${ }^{16}$, incluindo as etapas de pré-análise, exploração do material e tratamento dos resultados. Dessa forma, os principais aspectos de cada artigo selecionado foram colocados em uma tabela, para a realização de uma análise crítica dos parâmetros avaliados e dos resultados contidos neles.

\section{Resultados e Discussão}

Por meio de uma busca com a união dos termos prenatal stress e programming, foram encontrados 84 artigos, sendo selecionados 7. Quando utilizados os termos prenatal stress e pregnancy foram encontrados 208 artigos e, destes, 25 foram incluídos. Dos 292 artigos pesquisados com os termos citados anteriormente, 67 foram excluídos por serem revisões e outros 193 não foram incluídos por não estudarem o tema em questáo. Ao todo, 32 artigos foram selecionados para esta revisão. Os principais resultados foram sumarizados na Tabela 1.

De acordo com os critérios de seleção utilizados, todos os estudos selecionados foram realizados com roedores, sendo que a maioria foi em ratos 29/32 (91\%), e o restante 3/32 (9\%) em camundongos. Quanto à linhagem dos animais, nos estudos com ratos, 18 eram com Sprague-Dawley ${ }^{11,12,17-32}$ e 11 com Wistar ${ }^{10,13,14,33-40}$. Já nos estudos com camundongos, todos eram da linhagem Swiss ${ }^{9,15,41}$. Em relação ao sexo dos animais, a maioria dos estudos $(56 \%)$ avaliou machos e fême-


te em machos $15,17,19,28,29,33,36,37,39-41$ e $9 \%$ somente com fêmeas ${ }^{12,18,34}$. Quanto ao tipo de estresse, seguindo os critérios de inclusão, todos utilizaram contenção, sendo que $22 \%$ destes, além da contenção, utilizaram uma associação com iluminação específica feita por lâmpa$\mathrm{da}^{10,20,26,32,37-39}$. O estresse com frequência de três vezes ao dia foi o mais utilizado $(21)^{9,11-13,17-20,22-29,32,33,36,38,40}$, seguido de uma vez ao dia $(8)^{10,14,15,30,31,37,39,41}$, duas vezes ao dia $(2)^{21,35}$ e quatro vezes ao dia $(1)^{34}$. O tempo de duração do estresse também variou entre os artigos selecionados, com períodos de tempo compreendidos entre 30 minutos até 6 horas. $\mathrm{O}$ tempo de 45 minutos foi a duração escolhida pela grande maioria dos trabalhos $(62 \%)$.

Em relação ao período da gestação em que o estresse foi inserido, foram encontrados estudos na primeira, segunda e terceira semanas, assim como durante todo o período gestacional. O período mais utilizado para a inserção do estresse foi o da última semana, com $72 \%{ }^{9-}$ 14,18-23,25-27,30,31,33,34,36,37,39,40. Os diferentes períodos gestacionais são de suma importância para o desenvolvimento do filhote, tendo em vista que durante esses períodos ocorre o desenvolvimento e a maturação de diversos órgãos e sistemas ${ }^{20,33,42}$. Por exemplo, diferentes tipos de estresse durante a gestaçáo afetam o desenvolvimento do SNC de forma particular, dependendo do momento em que ocorrem no período gestacional, sendo mais grave e causando maiores alteraçóes no SNC quando o estresse acontece no período da segunda semana da gestação $^{42}$. Já em relação ao desenvolvimento pulmonar, em roedores inicia-se aproximadamente no $9^{\circ}$ dia de gestação e divide-se em quatro estágios: pseudoglandular (inicia do $9^{\circ}$ ao $16^{\circ}$ dia de gestação), canicular $\left(16^{\circ}\right.$ ao $17^{\circ}$ dia da gestação), sacular ( $17^{\circ}$ dia de gestação até o $5^{\circ}$ de vida do filhote) e alveolar ( $5^{\circ}$ dia de vida até o $\left.30^{\circ}\right)^{43}$. No entanto, os possíveis efeitos do estresse nestes períodos ainda não são conhecidos.

Muitos desfechos foram estudados nos diferentes artigos selecionados. Diversos estudos $(40 \%)^{11,17,22-}$ $25,27,33,34,36,37,40,41$ analisaram os efeitos do estresse durante o período gestacional no desenvolvimento ou na alteração de alguma estrutura no SNC. Entre estes, muitos analisaram áreas específicas, tais como, hipocampo, hipotálamo e mesencéfalo. Ainda, outros (9\%) também analisaram a resposta do eixo HPA ao estresse ${ }^{10,13,18}$, assim como os efeitos do estresse durante a gestaçáo no comportamento, aprendizado, medo e ansiedade (31\%) 9,10,12,14,15,29-31,38,39. Além disso, também há estu$\operatorname{dos}^{19-21,26,28}(15 \%)$ que analisaram os efeitos do estresse pré-natal em diferentes sistemas, como o endócrino, o cardiovascular e o musculoesquelético.

Ao analisar o comportamento de medo e ansiedade, os estudos que utilizaram estresse durante o período gestacional ainda são controversos. Um estudo realizado em camundongos machos adultos ${ }^{15}$ mostrou que o estresse durante a gestação causa um aumento dos níveis de medo e ansiedade, mostrando uma hipolocomoção na análise feita com os testes comportamentais de labirinto em cruz elevada e campo aberto. Da mesma forma, em ratos machos e fêmeas ${ }^{14}$, o estresse no período gestacional induz o aumento da ansiedade nas fêmeas e causa déficit de aprendizagem nos machos, demonstrando que as diferenças dos efeitos do estresse podem depender da severidade e do período da gestação. Porém, em outro estudo ${ }^{9}$ realizado em camundongos machos e fêmeas, o estresse pré-natal melhora o desempenho motor dos animais; no entanto, não houve alteração nos parâmetros de ansiedade. Já em outro estudo com ratos machos $^{39}$, o estresse durante a gestação diminuiu os níveis de medo e ansiedade, expressados por meio de uma maior locomoção, número de entradas e tempo nos braços abertos na análise comportamental.

Estudos que analisaram distúrbios hemodinâmicos em ratos machos e fêmeas ${ }^{20,26}$ constataram que o estresse no período gestacional pode afetar o desenvolvimento e a maturação de órgãos específicos relacionados ao controle da pressão, como coração, vasos, rins 
Tabela 1: Principais resultados encontrados nos artigos selecionados

\begin{tabular}{|c|c|c|}
\hline & $\begin{array}{c}\text { Número } \\
\text { de Artigos }\end{array}$ & $\begin{array}{c}\% \\
\text { (percentual) }\end{array}$ \\
\hline \multicolumn{3}{|l|}{ Espécies: } \\
\hline Ratos & 29 & 91 \\
\hline Camundongos & 3 & 9 \\
\hline \multicolumn{3}{|l|}{ Linhagem: } \\
\hline Sprague-Dawley & 18 & 57 \\
\hline Swiss & 3 & 9 \\
\hline Wistar & 11 & 34 \\
\hline \multicolumn{3}{|l|}{ Sexo: } \\
\hline Machos e Fêmeas & 18 & 57 \\
\hline Machos & 11 & 35 \\
\hline Fêmeas & 3 & 9 \\
\hline \multicolumn{3}{|l|}{ Tipos de Estresse: } \\
\hline Contenção & 25 & 78 \\
\hline Contenção+Luz brilhante & 7 & 22 \\
\hline \multicolumn{3}{|l|}{ Frequência: } \\
\hline 1 vez ao dia & 8 & 25 \\
\hline 2 vezes ao dia & 2 & 6 \\
\hline 3 vezes ao dia & 21 & 66 \\
\hline 4 vezes ao dia & 1 & 3 \\
\hline \multicolumn{3}{|l|}{ Tempo do Estresse: } \\
\hline 45 minutos & 20 & 63 \\
\hline 30 min a 1 hora & 12 & 37 \\
\hline \multicolumn{3}{|l|}{ Período do Estresse: } \\
\hline $2^{\mathrm{a}}$ semana & 9 & 28 \\
\hline $3^{\mathrm{a}}$ semana & 23 & 72 \\
\hline \multicolumn{3}{|l|}{ Desfechos: } \\
\hline SNC & 23 & 40 \\
\hline Eixo HРA & 3 & 9 \\
\hline $\begin{array}{l}\text { Comportamento/Aprendizagem/ } \\
\text { Medo/Ansiedade }\end{array}$ & 10 & 31 \\
\hline Outros sistemas & 4 & 15 \\
\hline
\end{tabular}

e cérebro. Neste modelo, os animais, quando expostos novamente ao estresse por contençáo quando adultos, não conseguem lidar de forma eficaz com a situaçáo, e apresentam aumento e variabilidade na pressão arterial, taquicardia e hipertensão induzida pelo estresse pré-natal.

Quando analisados memória e aprendizado, um estudo $^{30}$ mostrou que animais (de ambos os sexos) submetidos ao estresse durante a gestaçáo sofrem atraso na aprendizagem e prejuízos que afetam as memórias de curto e longo prazo, pois levam mais tempo para realizar algumas tarefas no campo de treino. Além disso, fêmeas expostas ao estresse no período gestacional possuem aprendizagem espacial prejudicada quando analisadas 24 meses depois. Da mesma forma, os machos tiveram diminuição da memória espacial nas atividades de reconhecimento espontâneo ${ }^{18}$.
Estudos que analisaram a resposta do eixo HPA apontam efeitos duradouros que causam aumento da reatividade em diferentes níveis deste eixo. Um estudo ${ }^{18}$ em ratas mostrou que há aumento da responsividade do eixo HPA como consequência do estresse na gestação, sendo que pode persistir até a senescência destas fêmeas. Ainda há uma correlação da hiper-reatividade do eixo HPA com problemas cognitivos e o envelhecimento. Outro estudo ${ }^{13}$ realizado com ratos machos e fêmeas, também demonstrou que a responsividade do eixo HPA está aumentada. Os resultados apresentados mostram aumento da concentração de corticosterona, hormônio adrenocorticotrófico (ACTH) e da expressão do RNAm do hormônio liberador de corticotrofina $(\mathrm{CRH})$, em resposta a um novo estresse na vida adulta. Esses efeitos dependem do sexo, fazendo que alteraçóes específicas em diferentes níveis do eixo tenham sido demonstradas para machos e fêmeas.

\section{Considerações finais}

Os resultados analisados nesta revisão indicam que o estresse no período gestacional provoca alteraçóes permanentes ao longo da vida em diversos sistemas. Estes efeitos parecem ser mais importantes no SNC e no controle dos mecanismos que regulam comportamentos de medo e ansiedade, assim como funções relacionadas à memória e aprendizagem. O período gestacional em que o estresse é inserido parece ser importante na definição dessas alteraçôes, assim como parece bem estabelecido que há diferenças nestas respostas entre machos e fêmeas. Um melhor entendimento de como situaçóes adversas no período gestacional influenciam o desenvolvimento e a resposta de importantes sistemas pode ajudar a prevençáo e o tratamento das consequências oriundas do estresse nesse período em seres humanos.

\section{Referências}

1. Chrousos GP. Stressors, stress, and neuroendocrine integration of the adaptive response: The 1997 Hans Selye Memorial Lecture. Annals of the New York Academy of Sciences. 1998; 851(1):311-35.

2. Wright RJ. Stress and atopic disorders. J Allergy Clin Immunol. 2005; 116(6):1301-6.

3. Glover V, O'Connor TG, O’Donnell K. Prenatal stress and the programming of the HPA axis. Neurosci Biobehav Rev. 2010; 35(1):17-22.

4. Holmes MC, Abrahamsen CT, French KL, Paterson JM, Mullins JJ, Seckl JR. The mother or the fetus? 11beta-hydroxysteroid dehydrogenase type 2 null mice provide 
evidence for direct fetal programming of behavior by endogenous glucocorticoids. J Neurosci. 2006; 26(14):3840-4.

5. Matthews SG. Antenatal glucocorticoids and programming of the developing CNS. Pediatr Res. 2000; 47(3):291-300.

6. Hauser J, Feldon J, Pryce CR. Direct and dam-mediated effects of prenatal dexamethasone on emotionality, cognition and HPA axis in adult Wistar rats. Horm Behav. 2009; 56(4):364-75.

7. Hodyl NA, Walker FR, Krivanek KM, Clifton V, Hodgson DM. Modelling prenatal bacterial infection: functional consequences of altered hypothalamic pituitary adrenal axis development. Behav Brain Res. 2007; 178(1):108-14.

8. Wright RJ. Prenatal maternal stress and early caregiving experiences: implications for childhood asthma risk. Paediatr Perinat Epidemiol. 2007; 21 [Suppl] 3:8-14.

9. Pallares ME, Scacchi Bernasconi PA, Feleder C, Cutrera RA. Effects of prenatal stress on motor performance and anxiety behavior in Swiss mice. Physiol Behav. 2007; 92(5):951-6.

10. Ordyan NE, Pivina SG. Characteristics of the behavior and stress-reactivity of the hypophyseal-adrenal system in prenatally stressed rats. Neurosci Behav Physiol. 2004; 34(6):569-74.

11. Bowman RE, MacLusky NJ, Sarmiento Y, Frankfurt M, Gordon M, Luine VN. Sexually dimorphic effects of prenatal stress on cognition, hormonal responses, and central neurotransmitters. Endocrinology. 2004; 145(8):3778-87.

12. Louvart H, Maccari S, Darnaudery M. Prenatal stress affects behavioral reactivity to an intense stress in adult female rats. Brain Res. 2005; 1031(1):67-73.

13. Garcia-Caceres C, Lagunas N, Calmarza-Font I, Azcoitia I, Diz-Chaves Y, Garcia-Segura LM, et al. Gender differences in the long-term effects of chronic prenatal stress on the HPA axis and hypothalamic structure in rats. Psychoneuroendocrinology. 2010; 35(10):1525-35.

14. Zagron G, Weinstock M. Maternal adrenal hormone secretion mediates behavioural alterations induced by prenatal stress in male and female rats. Behav Brain Res. 2006; 175(2):323-8.

15. Miyagawa K, Tsuji M, Fujimori K, Saito Y, Takeda H. Prenatal stress induces anxiety-like behavior together with the disruption of central serotonin neurons in mice. Neurosci Res. 2011; 70(1):111-7.

16. Bardin L, Reto LA, Pinheiro A. Análise de conteúdo: Ediçốes 70, Lisboa; 1979.

17. Mairesse J, Vercoutter-Edouart AS, Marrocco J, Zuena AR, Giovine A, Nicoletti F, et al. Proteomic characterization in the hippocampus of prenatally stressed rats. J Proteomics. 2012; 75(6):1764-70.

18. Darnaudery M, Perez-Martin M, Belizaire G, Maccari S, Garcia-Segura LM. Insulin-like growth factor 1 reduces age-related disorders induced by prenatal stress in female rats. Neurobiol Aging. 2006; 27(1):119-27.

19. Lesage J, Del-Favero F, Leonhardt M, Louvart H, Maccari $\mathrm{S}$, Vieau D, et al. Prenatal stress induces intrauterine growth restriction and programmes glucose intolerance and feeding behaviour disturbances in the aged rat. J Endocrinol. 2004; 181(2):291-6.

20. Igosheva N, Klimova O, Anishchenko T, Glover V. Prenatal stress alters cardiovascular responses in adult rats. J Physiol. 2004; 557(Pt 1):273-85.

21. Yum MS, Chachua T, Veliskova J, Velisek L. Prenatal stress promotes development of spasms in infant rats. Epilepsia. 2012; 53(3):e46-9.

22. Yeh CM, Huang CC, Hsu KS. Prenatal stress alters hippocampal synaptic plasticity in young rat offspring through preventing the proteolytic conversion of pro-brain-derived neurotrophic factor (BDNF) to mature BDNF. J Physiol. 2012; 590(Pt 4):991-1010.

23. Cai Q, Zhang B, Huang S, Wang T, Zhou T. The effects of prenatal stress on expression of CaMK-II and L-Ca2+ channel in offspring hippocampus. Acta Biochim Biophys Sin (Shanghai). 2011; 43(8):601-6.

24. Zuena AR, Mairesse J, Casolini P, Cinque C, Alema GS, Morley-Fletcher $S$, et al. Prenatal restraint stress generates two distinct behavioral and neurochemical profiles in male and female rats. PLoS One. 2008; 3(5):e2170.

25. Cai Q, Zhu Z, Huang S, Li H, Fan X, Jia N, et al. Sex and region difference of the expression of ERK in prenatal stress offspring hippocampus. Int J Dev Neurosci. 2007; 25(4):207-13.

26. Igosheva N, Taylor PD, Poston L, Glover V. Prenatal stress in the rat results in increased blood pressure responsiveness to stress and enhanced arterial reactivity to neuropeptide $\mathrm{Y}$ in adulthood. J Physiol. 2007; 582(Pt 2):665-74.

27. Cai Q, Zhu Z, Li H, Fan X, Jia N, Bai Z, et al. Prenatal stress on the kinetic properties of $\mathrm{Ca} 2+$ and $\mathrm{K}+$ channels in offspring hippocampal CA3 pyramidal neurons. Life Sci. 2007; 80(7):681-9.

28. D'Mello A P, Liu Y. Effects of maternal immobilization stress on birth weight and glucose homeostasis in the offspring. Psychoneuroendocrinology. 2006; 31(3):395-406.

29. Laviola G, Rea M, Morley-Fletcher S, Di Carlo S, Bacosi A, De Simone R, et al. Beneficial effects of enriched environment on adolescent rats from stressed pregnancies. Eur J Neurosci. 2004; 20(6):1655-64.

30. Meunier J, Gue M, Recasens M, Maurice T. Attenuation by a sigma1 (sigma1) receptor agonist of the learning and memory deficits induced by a prenatal restraint stress in juvenile rats. Br J Pharmacol. 2004; 142(4):689-700.

31. Gue M, Bravard A, Meunier J, Veyrier R, Gaillet S, Recasens M, et al. Sex differences in learning deficits induced by prenatal stress in juvenile rats. Behav Brain Res. 2004; 150(1-2):149-57.

32. Edwards HE, Dortok D, Tam J, Won D, Burnham WM. Prenatal stress alters seizure thresholds and the development of kindled seizures in infant and adult rats. Horm Behav. 2002; 42(4):437-47.

33. Baquedano E, Garcia-Caceres C, Diz-Chaves Y, Lagunas N, Calmarza-Font I, Azcoitia I, et al. Prenatal stress induces 
long-term effects in cell turnover in the hippocampus-hypothalamus-pituitary axis in adult male rats. PLoS One. 2011; 6(11):e27549.

34. Souza MA, Szawka RE, Centenaro LA, Diehl LA, Lucion AB. Prenatal stress produces sex differences in nest odor preference. Physiol Behav. 2012; 105(3):850-5.

35. Sadaghiani MM, Saboory E. Prenatal stress potentiates pilocarpine-induced epileptic behaviors in infant rats both time and sex dependently. Epilepsy Behav. 2010; 18(3):166-70.

36. Carboni E, Barros VG, Ibba M, Silvagni A, Mura C, Antonelli MC. Prenatal restraint stress: an in vivo microdialysis study on catecholamine release in the rat prefrontal cortex. Neuroscience. 2010; 168(1):156-66.

37. Ordyan NE, Pivina SG. Effects of prenatal stress on the activity of an enzyme involved in neurosteroid synthesis during the "critical period" of sexual differentiation of the brain in male rats. Neurosci Behav Physiol. 2005; 35(9):931-5.
38. Bethus I, Lemaire V, Lhomme M, Goodall G. Does prenatal stress affect latent inhibition? It depends on the gender. Behav Brain Res. 2005; 158(2):331-8.

39. Ordyan NE, Pivina SG. Anxiety levels and neurosteroid synthesis in the brains of prenatally stressed male rats. Neurosci Behav Physiol. 2003; 33(9):899-903.

40. Berger MA, Barros VG, Sarchi MI, Tarazi FI, Antonelli MC. Long-term effects of prenatal stress on dopamine and glutamate receptors in adult rat brain. Neurochem Res. $2002 ; 27(11): 1525-33$.

41. Son GH, Chung S, Geum D, Kang SS, Choi WS, Kim K, et al. Hyperactivity and alteration of the midbrain dopaminergic system in maternally stressed male mice offspring. Biochem Biophys Res Commun. 2007; 352(3):823-9.

42. Meyer U, Yee BK, Feldon J. The neurodevelopmental impact of prenatal infections at different times of pregnancy: the earlier the worse? Neuroscientist. 2007; 13(3):241-56.

43. Warburton D, Schwarz M, Tefft D, Flores-Delgado G, Anderson KD, Cardoso WV. The molecular basis of lung morphogenesis. Mech Dev. 2000; 92(1):55-81. 